\title{
Simulations on many scales: The synapse as an example*
}

\author{
Kaihsu Tai ${ }^{\ddagger}$ \\ Department of Biochemistry, University of Oxford, Rex Richards Building, South \\ Parks Road, Oxford OX1 3QU, UK
}

\begin{abstract}
Computer simulation methods spanning several temporal and spatial scales are reviewed, focusing on their applications on the neuromuscular synapse. Quantum mechanics treats the enzymatic catalysis of neurotransmitters on the picometer scale. Molecular dynamics reveals conformational changes of the enzyme acetylcholinesterase for nanoseconds. Brownian dynamics follow the substrate molecule in its diffusion on the microsecond level. Methods such as finite elements describe the diffusion of neurotransmitters as a changing concentration continuum in the synapse. Promising directions for future research include integration of methods on several scales, and applying these methods to the acetylcholine receptor.
\end{abstract}

\section{OVERVIEW}

With the development of both theory and computer technology, simulation has become a viable way of investigating the behavior of molecules. A series of simulation methods are now available to address chemical questions at several time and length scales [1]. The success of these methods in "pure", theoretical chemistry has been matched by their applications in materials science.

Biochemistry, the molecule-based study of life, also requires understanding on several scales, some of which are not readily reached by experiment. Encouragingly, it is possible to apply the simulation methods in biochemistry (Fig. 1, p. 298). Here, some of these endeavors-as applied on an example, the synapse in the nervous system - are discussed, after a short presentation of several simulation methods for different temporal and spatial scales.

Quantum mechanics (QM) calculations have been firmly established as a rigorous methodology; their pioneers were awarded a Nobel Prize in chemistry in 1998 [2,3]. Combined with larger-grain methods such as molecular dynamics (see below), treatment of enzymatic catalysis to a degree comparable to that for small-molecule reactions is feasible.

First applied to proteins more than 25 years ago by McCammon and coworkers [4], the method of Newtonian molecular dynamics (MD) has now entered the mainstream of biochemistry [5]. MD aptly reveals the macromolecular conformational changes on the nanosecond-level, filling a time-resolution gap (until recently) long left by experimental methods.

The methods MD, Brownian dynamics (BD) [6], Monte Carlo (MC) simulation, and the finite elements (FE) [7] together offer a spectrum of tools, describing diffusion either in a discrete fashionmolecule-by-molecule - or as a continuum of concentration. Recently, other mesoscale and multiscale methods have emerged, with the promise of bringing up the scale of simulations to the cellular level. Ambitious projects aim at yet larger scales, but they lie beyond the scope of this review $[8,9]$.

*Pure Appl. Chem. 76, 263-319 (2004). A collection of invited, peer-reviewed articles by the winners of the 2003 IUPAC Prize for Young Chemists.

${ }^{\dagger}$ Fax: +44 1865 275182; E-mail: kaihsu@biop.ox.ac.uk 


\section{NEUROTRANSMITTER DIFFUSION IN THE SYNAPSE: FINITE-ELEMENT AND MONTE CARLO SIMULATIONS}

The synapse is the point of communication where a neuron sends its signal to another cell. There are two types of synapses, categorized by the manner of transmission: electrical synapses and chemical synapses [10-12]. The discussion here focuses on the neuromuscular junction (NMJ), the chemical synapse between the neuron and the muscle cell. The geometries of the synaptic cleft, bounded by preand postsynaptic membranes, differ by muscle type.

Synaptic signal can be carried by the neurotransmitter acetylcholine (ACh) [13], which is released by the neuron upon arrival of an action potential. ACh diffuses across the cleft, binds to and activates the postsynaptic acetylcholine receptors (AChR), generating a response in the receiving cell. The enzyme acetylcholinesterase (AChE) quickly hydrolyzes ACh to acetate and choline, deactivating the receptor and tapering off the response.

This diffusion process of neurotransmitters in the synapse has been simulated by solving differential equations governing the diffusion-reaction of the neurotransmitter concentration seen as a continuum using FE [14-16]; or by tracking the diffusion-reaction of each neurotransmitter molecule using MC simulations [17,18].

MC simulations model the neurotransmitter diffusion by carrying out grid-free (that is, undiscretized) 3-dimensional random walks of each molecule. During the random walk, the molecule encounters surfaces and effector (receptor and esterase) sites. The outcome of encounter is determined by MC probablistic criteria for the acceptance or rejection of a proposed step. The parameters are set to fit analytical results of diffusion and reaction processes.

The FE method, on the other hand, discretizes the 3-dimensional space but sees the neurotransmitter as continuous concentration. At each vertex of the discretized space sits a simple function-an element-to represent the value of the neurotransmitter concentration. The diffusion equation is solved in time over this spatial domain to represent the movement of this concentration.

Both methods have been improved to accommodate geometries coming straight from 3-dimensional electron tomography $[19,20]$ rather than simple, rectangular models, affording simulations up to the millisecond scale. At the moment, the FE models are not as mature as Monte Carlo ones in representing all the components in synaptic transmission, and thus may not fit the experimental results as well. However, to reflect muscle contraction, changing spatial domain is possible in FE. Therefore, efforts in integrating these two methods will be worthwhile.

\section{DIFFUSION OF THE SUBSTRATE TO THE ENZYME: BROWNIAN DYNAMICS}

Brownian dynamics uses a stochastic algorithm to simulate the diffusion of a substrate molecule under the influence of an enzyme. Consider how a substrate molecule gets to the enzyme molecule: At infinite separation, the interaction between the two molecules is nearly isotropic and negligible; the diffusion of the substrate area relates only to the diffusion coefficient- the reaction rate due to this "far" part may be analytically obtained. Upon close approach, the interaction becomes significant in addition to the Brownian, stochastic motions; it is at such a "begin surface" that the enzyme imposes its electrostatics on the substrate in BD simulations. Observing the odds of the substrate succeeding in being catalyzed by the enzyme against that of it diffusing away to a "quit surface" (a stand-in for infinite separation), the reaction rate due to the close approach may be found. Multiplying the analytical "far" rate with this "near" rate, BD can be used to obtain reaction coefficients comparable to those obtained from experiments [6].

This method has been used to explain the electrostatic effects of mutant AChE observed in experiment [21]. The kinetic observations can be reproduced at the microsecond scale, even when a peptidic snake-toxin inhibitor is involved [22]. (See below for more BD applications.) 


\section{CONFORMATIONAL CHANGES IN ACETYLCHOLINESTERASE: MOLECULAR DYNAMICS}

\section{Dynamical selectivity at the gorge}

The enzyme $\mathrm{AChE}$ has several intriguing qualities. First, its key responsibility for regulating synaptic transmission has made it a target of several chemical agents: from the drugs countering Alzheimer's disease [23,24] and myasthenia gravis (serious muscle weakness) [25], to snake toxins and chemical weapons (such as sarin and VX) [26]. Second, the active site of AChE is buried $2 \mathrm{~nm}$ deep in the center of the enzyme, and connected to the protein surface by a narrow "gorge" [27]. This design is counterintuitive, considering the rapid catalysis of AChE; however, it has been suggested that selective control of substrate entry may be facilitated by a dynamical, fluctuating gorge $[28,29]$.

In the static crystallographic structure, fasciculin obstructs the entrance to the gorge, but it does not reach down the gorge. Two MD simulations have been performed from this structure, each amounting to several nanoseconds: one of AChE by itself [30-32], the other of AChE with a snake toxin, fasciculin $[33,34]$.

\section{Mechanisms of AChE inhibition by fasciculin}

Measuring the width ("proper radius") of the gorge every picosecond in the simulations, a histogram can be made of its distribution [35]. Strikingly, this is not simply a symmetric, Gaussian distribution; there are two substates, one wider and one narrower. In addition, fasciculin shifts the whole distribution toward lower width values, and favors the narrower substate. These observations suggest that fasciculin restricts AChE gorge fluctuation in a dynamical fashion.

The average structures from the two simulations showed that the active site of AChE was changed: the histidine residue that served as the bridge in the proton-transfer pathway during catalysis has been oriented away from its normal position. As fasciculin did not reach down to the active site, this disruption of the active site could only happen by allosteric means.

In summary, fasciculin inhibits AChE by three mechanisms: (i) steric obstruction of the entrance of the gorge; (ii) dynamic restriction of gorge width; and (iii) allosteric disruption of the active site conformation. The first is a direct observation from the static crystallographic structure; the latter two are observations from the simulations.

\section{"Porcupine plots": Visualizing concerted motions in AChE gorge fluctuation}

What controls the fluctuation of the AChE gorge? Is it merely the movements of a few residues around the gorge bottleneck, or are there more global motions at work? Without an analytical tool at hand, it is difficult to sort through the large amount of data from the simulations in order to determine one way or the other. A simple device-the porcupine plot—can help us understand the correlation of motion in different parts of the protein with a functionally important motion, the fluctuation of the gorge width in this case.

By plotting the correlation vector between the movement of each $\alpha$-carbon atom and the gorge width, one sees the likelihood and direction of concerted motions of different parts of the protein whenever the gorge becomes open. The viewer looks down the gorge in the figures discussed below. In Fig. 2, the gorge opening not only involves the residues near the gorge, but even residues some distance from the gorge also move concertedly to make way for the gorge to open. With fasciculin bound (Fig. 3), much of this coordination has been suppressed; this may be one of the mechanisms by which fasciculin restricts the opening of the gorge. The simple device of porcupine plot has proven to be useful in visualizing the concerted motions in proteins. 
These nanosecond-scale simulations have enabled us to make comparisons with experiments. Fluorescence anisotropy decay experiment, a method detecting fast motions in proteins, has the resolution of nanoseconds. The simulation results has been confirmed by comparing the decay of anisotropy due to protein segmental motions calculated from simulation and that observed from experiment [36].

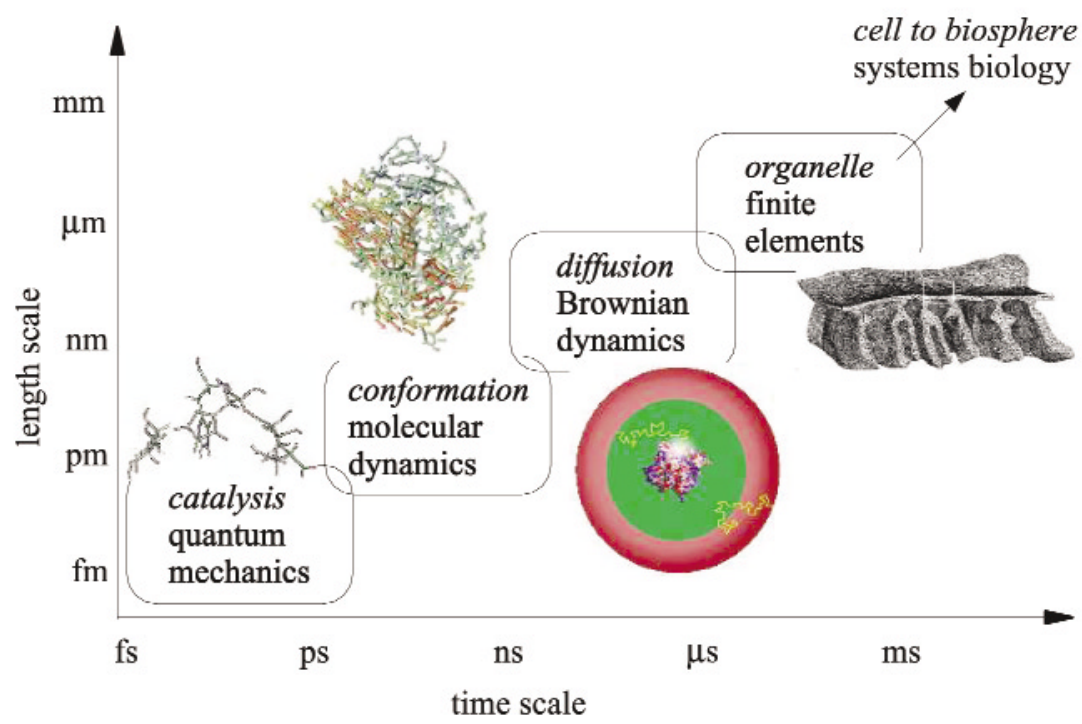

Fig. 1 Multiscale modeling as applied to biomolecular systems; after [1]. The inset images illustrate the scale each modeling method covers (courtesy of the McCammon Group).

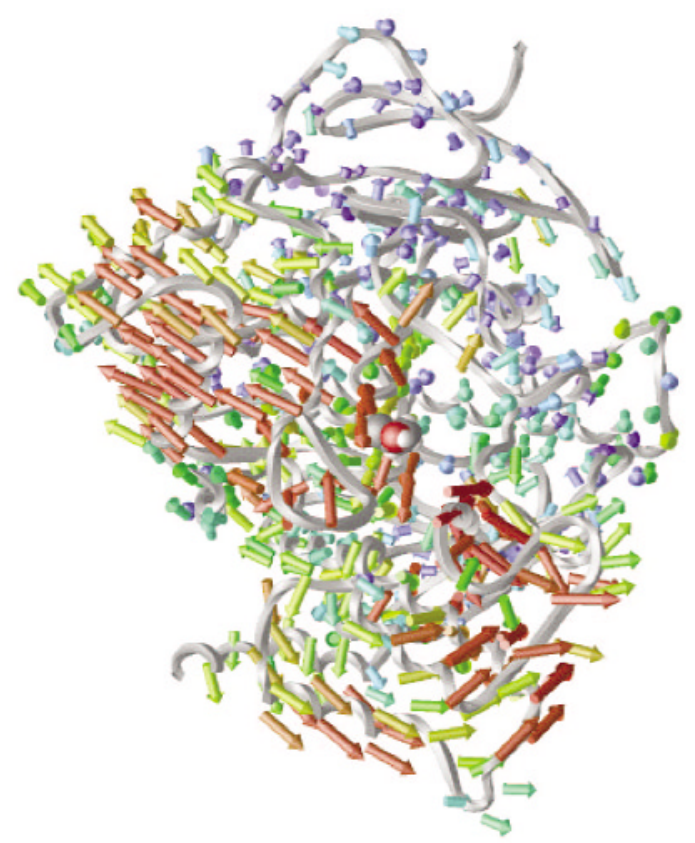

Fig. 2 Porcupine plot from the simulation of AChE by itself. The color of each vector corresponds to the magnitude thereof. 


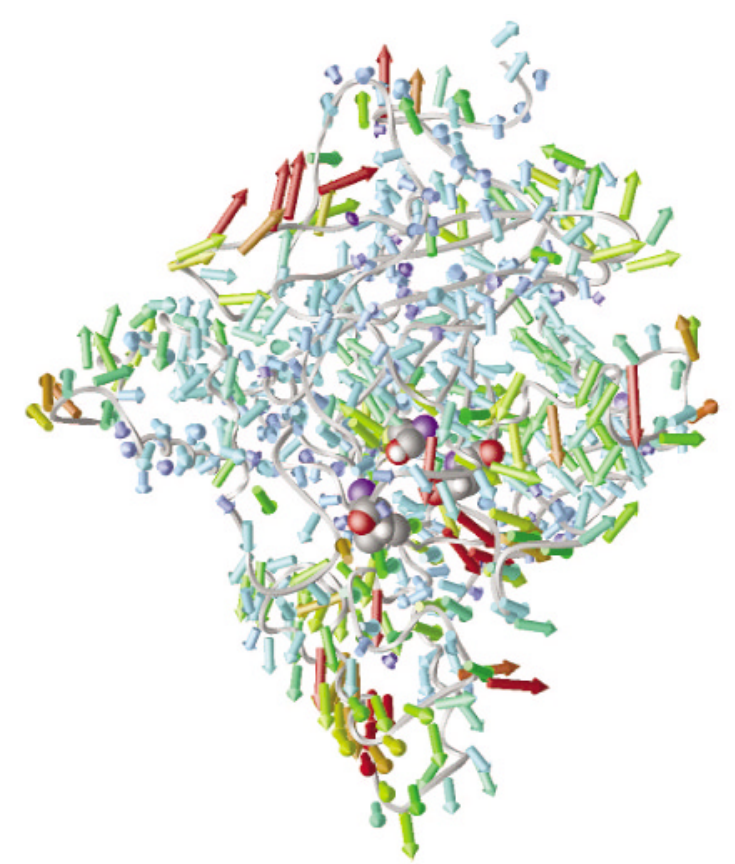

Fig. 3 Porcupine plot from that of AChE, when fasciculin (not shown) is bound. The color of each vector corresponds to the magnitude thereof.

\section{Docking studies}

After performing MD, it is possible to dock ligands to several snapshots (instantaneous conformations of the molecule) obtained therefrom; this is called the multiple docking method. Several ligands were docked to snapshots from MD trajectories of unliganded and ACh-bound AChE [37]. Kinetic coefficients from experiments were reproduced from docking energies. In addition, it is found that ACh stabilizes the catalytic triad and makes ligand binding even more favorable.

Catalysis, enzymatic or not, is based on the lowering of activation energy. Mutants can easily be made in simulation to understand better the basis of catalysis in AChE [38]. Extending previous findings, perturbations that decrease steric complementarity affect binding unfavorably. One essential advantage of such simulations is the ability to test theories where experiments are not readily feasible: for example, "turning off" electrostatic charges; testing a sizeable collection mutant-ligand pairs.

\section{Movements of water}

At this point, it is appropriate to review the sequence of events occurring to the substrate $\mathrm{ACh}$, and the relevant simulation methods. Starting some distance away from AChE up to its surface, the long-distance diffusion is covered by FE and BD simulations. From there to the active site, the close contact is covered by MD. In FE and BD, water is treated with bulk parameters; in MD, the position each water molecule can be explicitly tracked. MD reasonably reproduces the bulk properties of water, providing a connection between the scales. Furthermore, sites frequented by water molecules on the surface of $\mathrm{AChE}$ have been identified; the movements of water molecules among the sites and into bulk were measured [39].

Once ACh gets to the surface of AChE, it has to go down the gorge. At this point, the effects of water molecules in the gorge-rather than in the bulk-become prominent: they have to "get out of the way" for ACh to enter, and for the product of hydrolysis to escape from the gorge. In the MD simula- 
tions, fluctuations in the number of the gorge water molecules, and the occurrence of transient cavities points to processes that aid substrate entrance and product escape [40].

\section{Ligand crossing the barrier}

The MD method can be extended to investigate phenomena of particular interest. Two such extensions have been carried out on the AChE gorge to probe the entrance and escape of ligands: one used steered MD, which pulls the ligand from the gorge by force, to model ligand escape [41]; the other uses umbrella sampling, which uses potential wells to restrict the ligand, to obtain better sampling of conformational changes in $\mathrm{AChE}$ as a ligand moves in the gorge [42]. The latter study provided a potential of mean force for the ligand in the gorge. The findings from these studies imply facile ligand movement in the gorge given changes in AChE conformation-local or global—the design of AChE does not seem to be so counterintuitive after all.

Recalling previous BD work $[21,22]$ including the simplified BD models explaining dynamical selectivity [28,29], a current BD study [43] attempts to apply the potential of mean force obtained from the umbrella-sampling MD, presenting a comprehensive picture of gorge dynamical selectivity.

\section{CATALYSIS AT THE ACTIVE SITE: QUANTUM MECHANICS}

Due to limits in computational power, enzyme systems are too large to treat in whole with QM calculation. It is encouraging that the mechanics that enabled MD may be mixed with QM to give "QM/MM" simulations: molecular mechanics (MM) takes care of the global conformational changes while QM treats the bond-making/bond-breaking processes at the active site, which is beyond the capability of MM. This mixed method has been used to confirm the experimentally observed acylation energy barrier for catalysis in AChE and the effect of an "oxyanion hole" formed by three residues in stabilizing high-energy transition states [44,45]; such investigations would be impossible with only conventional MD. It also showed the effects of global enzyme conformation on catalysis, highlighting the importance of MM in complementing QM calculations [46].

\section{CONCLUSION AND PROSPECTS}

Here, a series of simulations at different scales applied on molecules and processes in the synapse has been presented, with MD being the centerpiece from which this tour d'horizon extends. Note that at the moment the pieces in this tour have been done disjointly, though each endeavors to connect with its neighbors in the next scale. Reports of successful multiscale imaging have appeared [20,47]. The time may be ripe for synchronous multiscale simulations, where simulations of different scales run at the same time on computers and feed results as parameters into each other. A missing piece in simulating the synapse is AChR. Very recently, useful high-resolution structures thereof have become available [48-50]; this is another promising project [51].

\section{ACKNOWLEDGMENTS}

My sincere gratitude is directed to my doctoral advisor, Prof. J. Andrew McCammon, for his insight and guidance; to Dr. Hugh R. MacMillan for useful discussions; and to Ms. Jennifer M. Bui, Dr. Richard H. Henchman, Dr. Jeremy Kua, Dr. Alexei A. Podtelezhnikov, and Prof. Yingkai Zhang for sharing their unpublished results. I thank Prof. Mark S. P. Sansom, La Jolla Interfaces in Science training program, the Burroughs-Wellcome Fund, and the Biotechnology and Biological Sciences Research Council (UK) for support. 


\section{REFERENCES}

1. W. A. Goddard III, T. Cagin, M. Blanco, N. Vaidehi, S. Dasgupta, W. Floriano, M. Belmares, J. Kua, G. Zamanakos, S. Kashihara, M. Iotov, G. Gao. Comput. Theor. Polym. Sci. 11, 329-343 (2001).

2. J. Pople. "Quantum chemical models", in Nobel Lectures, Chemistry 1996-2000, World Scientific, Singapore (1998).

3. W. Kohn. "Electronic structure of matter: Wave functions and density functionals", in Nobel Lectures, Chemistry 1996-2000, World Scientific, Singapore (1999).

4. J. A. McCammon, B. R. Gelin, M. Karplus. Nature 267, 585-590 (1977).

5. M. Karplus and J. A. McCammon. Nat. Struct. Biol. 9, 646-652 (2002).

6. R. R. Gabdoulline and R. C. Wade. Curr. Opin. Struct. Biol. 12, 204-213 (2002).

7. D. Braess. Finite Elements, Cambridge University Press, Cambridge (1997).

8. H. Kitano. Nature 420, 206-210 (2002).

9. K. S. Brown and J. P. Sethna. Phys. Rev. E 68, 021904 (2003).

10. E. R. Kandel, J. H. Schwartz, T. M. Jessell. Principles of Neural Science, Appleton and Lange, Norwalk, CT (1991).

11. H. Zimmermann. Synaptic Transmission: Cellular and Molecular Basis, Thieme, Stuttgart (1993).

12. G. M. Shepherd. Neurobiology, Oxford University Press, Oxford (1994).

13. P. Taylor and J. H. Brown. "Acetylcholine", in Basic Neurochemistry: Molecular, Cellular and Medical Aspects, G. J. Siegel, B. W. Agranoff, R. W. Albers, S. K. Fisher, M. D. Uhler (Eds.), Lippincott Williams and Wilkins, Philadelphia (1999).

14. J. L. Smart and J. A. McCammon. Biophys. J. 75, 1679-1688 (1998).

15. T. Ghaffari-Farazi, J.-S. Liaw, T. W. Berger. Neurocomputing 26-27, 17-27 (1999).

16. K. Tai, S. D. Bond, H. R. MacMillan, N. A. Baker, M. J. Holst, J. A. McCammon. Biophys. J. 84, 2234-2241 (2003).

17. J. R. Stiles and T. M. Bartol. "Monte Carlo methods for simulating realistic synaptic microphysiology using Mcell”, in Computational Neuroscience: Realistic Modeling for Experimentalists, E. De Schutter (Ed.), CRC Press, New York (2000).

18. J. R. Stiles, T. M. Bartol, M. M. Salpeter, E. E. Salpeter, T. J. Sejnowski. "Synaptic variability: New insights from reconstructions and Monte Carlo simulations with MCell", in Synapses, W. M. Cowan, T. C. Südhof, C. F. Stevens (Eds.), Johns Hopkins University Press, Baltimore (2001).

19. M. L. Harlow, D. Ress, A. Stoschek, R. M. Marshall, U. J. McMahan. Nature 409, 479-484 (2001).

20. J. S. Gustafsson, A. Birinyi, J. Crum, M. Ellisman, L. Brodin, O. Shupliakov. J. Comp. Neurol. 450, 167-182 (2002).

21. S. Tara, A. H. Elcock, P. D. Kirchhoff, J. M. Briggs, Z. Radic, P. Taylor, J. A. McCammon. Biopolymers 46, 465-474 (1998).

22. A. H. Elcock, R. R. Gabdoulline, R. C. Wade, J. A. McCammon. J. Mol. Biol. 291, 149-162 (1999).

23. S. Tara, V. Helms, T. P. Straatsma, J. A. McCammon. Biopolymers 50, 347-359 (1999).

24. J. Grutzendler and J. C. Morris. Drugs 61, 41-52 (2001).

25. E. T. Wittbrodt. Arch. Intern. Med. 157, 399-408 (1997).

26. P. Taylor. "Anticholinesterase agents", in The Pharmacological Basis of Therapeutics, J. G. Hardman, L. E. Limbird, P. B. Molinoff, R.W. Ruddon, A. G. Gilman (Eds.), McGraw-Hill, New York (1996).

27. P. Marchot, Y. Bourne, C. N. Prowse, P. E. Bougis, P. Taylor. Toxicon 36, 1613-1622 (1998).

28. H.-X. Zhou, S. T. Wlodek, J. A. McCammon. Proc. Natl. Acad. Sci. USA 95, 9280-9283 (1998).

29. N. A. Baker and J. A. McCammon. J. Phys. Chem. B 103, 615-617 (1999). 
30. S. Tara, T. P. Straatsma, J. A. McCammon. Biopolymers 50, 35-43 (1999).

31. K. Tai, T. Shen, U. Börjesson, M. Philippopoulos, J. A. McCammon. Biophys. J. 81, 715-724 (2001).

32. T. Shen, K. Tai, R. Henchman, J. A. McCammon. Acc. Chem. Res. 35, 332-340 (2002).

33. K. Tai, T. Shen, R. H. Henchman, Y. Bourne, P. Marchot, J. A. McCammon. J. Am. Chem. Soc. 124, 6153-6161 (2002).

34. J. M. Bui, K. Tai, J. A. McCammon. To be submitted for publication.

35. T. Y. Shen, K. Tai, J. A. McCammon. Phys. Rev. E 63, 041902 (2001).

36. J. Shi, K. Tai, J. A. McCammon, P. Taylor, D. A. Johnson. J. Biol. Chem. 278, 30905-30911 (2003).

37. J. Kua, Y. Zhang, J. A. McCammon. J. Am. Chem. Soc. 124, 8260-8267 (2002).

38. J. Kua, Y. Zhang, A. C. Eslami, J. R. Butler, J. A. McCammon. Protein Sci. 12, 2675-2684 (2003). In press.

39. R. H. Henchman and J. A. McCammon. Protein Sci. 11, 2080-2090 (2002).

40. R. H. Henchman, K. Tai, T. Shen, J. A. McCammon. Biophys. J. 82, 2671-2682 (2002).

41. Y. Xu, J. Shen, X. Luo, I. Silman, J. L. Sussman, K. Chen, H. Jiang. J. Am. Chem. Soc. 125, 11340-11349 (2003).

42. J. M. Bui, R. H. Henchman, J. A. McCammon. Biophys. J. 85, 2267-2272 (2003).

43. A. A. Podtelezhnikov, J. Bui, J. A. McCammon. To be submitted for publication.

44. Y. Zhang, J. Kua, J. A. McCammon. J. Am. Chem. Soc. 124, 10572-10577 (2002).

45. M. M. Hurley, J. B. Wright, G. H. Lushington, W. E. White. Theor. Chem. Acc. 109, 160-168 (2003).

46. Y. Zhang, J. Kua, J. A. McCammon. J. Phys. Chem. B 107, 4459-4463 (2003).

47. R. D. Shoop, E. Esquenazi, N. Yamada, M. H. Ellisman, D. K. Berg. J. Neurosci. 22, 748-756 (2002).

48. A. Miyazawa, Y. Fujiyoshi, M. Stowell, N. Unwin. J. Mol. Biol. 288, 765-786 (1999).

49. A. Miyazawa, Y. Fujiyoshi, N. Unwin. Nature 423, 949-955 (2003).

50. Y. Paas, J. Cartaud, M. Recouvreur, R. Grailhe, V. Dufresne, E. Pebay-Peyroula, E. M. Landau, J.-P. Changeux. Proc. Natl. Acad. Sci. USA 100, 11309-11314 (2003).

51. R. Henchman, H.-L. Wang, S. Sine, P. Taylor, J. A. McCammon. Biophys. J. 85, 3007-3018 (2003). 